\title{
Highlights of Italian hospital pharmacy: experiences and research
}

\author{
Ilaria Uomo, ${ }^{1 *}$ Valeria Fadda, ${ }^{2 * *}$ Paolo Serra, ${ }^{3 *}$ Federica Locchi, ${ }^{4 * *}$ Francesco Cattel, ${ }^{5 *}$ Nicoletta Ambrogi, ${ }^{6 \$}$ \\ Francesca Venturini ${ }^{\circ \circ}$
}

The seminar will approach Italian hospital pharmacy from different views, presenting four highly relevant pharmacy practice issues in Italy: (1) postgraduate education (eg, specialisation in hospital pharmacy); (2) new professional models (eg, the ward pharmacist project); (3) continuity of care (eg, the role of the health system pharmacist); and (4) health technology assessment (eg, an example of a research project through the hospital pharmacists' network).

The current state of Italian hospital pharmacy practice may largely differ depending on the region and hospital size.

SIFO (Società Italiana di Farmacia Ospedaliera e dei Servizi Farmaceutici delle Aziende Sanitarie), the Italian Society of Health System Pharmacists, is a scientific association embracing almost all public pharmacists employed by the National Health Service. The society is particularly committed to creating a collaborative network of practising pharmacists, with different aims: research purposes, enhancement of professional skills and promotion of the education of young pharmacists.

During the session, speakers will underline some of these projects, presented by two facilitators: Francesca Venturini, chairman of the SIFO scientific committee and Nicoletta Ambrogi, head of the SIFO delegation of European Association of Hospital Pharmacists.

\section{THE NETWORK OF HOSPITAL} PHARMACY SPECIALISATION SCHOOLS

The first topic will cover the recently revised programme of the hospital pharmacy specialisation school, and will be explained by two pharmacists: Dr Paolo

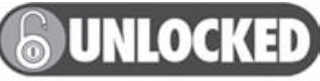

This paper is freely available online under the BMJ Journals unlocked scheme, see http:// ejhp.bmj.com/info/unlocked.dtl

Correspondence to Dr I Uomo,

Department of Pharmacy, ASP Palermo via Pindemonte 8890128 Palermo Italy.
Serra (SIFO national coordinator for the schools of specialisation) and Dr Federica Locchi (a specialising pharmacist at the University of Bologna).

The 2009 school reform greatly changed the content and structure of the specialisation school: the programme was extended to 4 years, and breakdown between the time spent at the practice sites (eg, hospital pharmacies and outpatient pharmaceutical departments) and at the university favoured the first, by a 70/30 ratio.

The postgraduate school student interacts at several levels: at the practice sites with the tutors and the hospital pharmacy director and at the university with the faculty and dean of the school of pharmacy.

In this context, it is essential to harmonise both university and practice programmes in order to ensure a homogeneous education all over the country. Moreover, close interaction between academics and practice is essential to ensure the multitask training aimed at educating an up to date hospital pharmacy specialist.

SIFO provides its support with the Scuole di Specializzazione in Farmacia Ospedaliera network, a web based tool that improves communication, cross talking and problem solving between directors, teachers, tutors and students of the different Italian schools. At present, five out of 23 schools are testing the platform (Bologna, Genova, Novara, Bari and Siena).

The main aims of the project are:

- To harmonise programmes across universities

- To harmonise practice content among practice sites

- To promote multi-site and multi-school projects.

The main tasks of the platform are:

- Sharing class programmes and teaching materials

- Cross alerts on seminar and national events

- Forum on specific topics

- Tool for multicentre trials

- 'Benchmark' in professional formation

- National map of schools, hospitals and sanitary structures involved in the formation.
This dynamic network is also exportable in other European realities.

\section{HEALTH TECHNOLOGY ASSESSMENT}

Dr Valeria Fadda will describe the Italian experiences with health technology assessment (HTA), a multidisciplinary field of policy analysis. SIFO, in the last 4 year programme, included HTA as one of the priority activities and it is supporting this important challenge for the development, diffusion and use of health technology in terms of economic outcomes also.

In recent times there has been a growing pressure to better manage healthcare expenditures and to balance opportunity costs and resource allocation. For this reason, HTA is increasingly being recognised in Italy as an essential tool for the decision making process, mostly at the regional level.

SIFO has long been engaged in the containment of healthcare expenditure by promoting both efficiency and quality.

High risk medical devices are becoming an increasingly important focus of interest for both the Italian and European scientific communities because of the low level of regulation in this field in the premarketing and postmarketing phases. The contribution of SIFO in this specific area is expressed through the 'across Italy project', a joint work among hospital pharmacists involved in evaluation of medical devices.

The aim of the project is to encourage economic and clinical evaluation of medical devices in order to ensure patient safety and allocate resources for safe and effective devices and, in addition, to share the evaluations with different hospitals.

The relevant items shared between the participating centres are mainly the local reimbursement price and a short evaluation report of the device. The form is a summary of the clinical, economic and general information about the device and it acts as an aid for local decision makers who are called on to decide on the governance of the specific device.

Currently, 20 centres joined the project and 43 evaluations have been made available. The names of the hospitals and evaluations are available (in Italian only) here: http://www.osservatorioinnovazione. net/acrossitaly.htm 
For 39 out of 43 evaluations, a governance proposal was suggested.

- Thirty one devices had weak clinical evidence so that the governance proposal was not to use the device.

- Five devices failed to show statistical superiority over the comparator(s) and were therefore handled through a cost minimisation analysis.

- Only one device was considered to be safe, effective and possibly cost effective, and approval was suggested.

- For two devices a payback registry institution was proposed.

\section{THE 'DEPARTMENT' PHARMACIST}

Dr Francesco Cattel will report on one of the most important projects conducted in 2010/2011 in our profession. In this project, the Italian Ministry of Health promoted a model, applied in five pilot hospitals, to enhance the value of the pharmacist in a multidisciplinary team as part of the patient care process, as a guarantee of prescription appropriateness and as an 'added value' in different areas of health intervention. In particular, a pharmacist was assigned to assist physicians and nurses in the oncology wards. There were five main indicators of the study: two were related to prescriptions (improving the quality of the data recorded in the Italian web based register on antineoplastic drugs and monitoring of off label prescriptions, called ONCO-AIFA); the third was on patient safety (eg, reporting of near misses); the fourth was on drug management; and the last was on patient perceived quality of the service rendered by the pharmacist. Improvement in all areas was demonstrated.

The results obtained from the indicators of the ministerial project showed:

Requirements to register in accordance with ONCO-AIFA: this web based register foresees that every clinician has to enter information on the patient's vital statistics, disease, drug schedule (first time) and then toxicity, variations on dosage, final outcome (follow-up). If all the elements match the required parameters, the prescription is allowed and the hospital pharmacist can dispense the drug. Standard reports are then made available on a local (hospital), regional and national basis. This study showed an increased number of patients included ( $71.7 \%$ on average), increased number of cards closed (over 600\%), increase in revaluation of patients enrolled (69.8\% on average), increased dispensation numbers (166.7\% on average) and increased number of services that conformed to the register (78.7\%).

Reduction in the value of Euro stocks of department of medicines in the closet: reducing stocks in Euro (-49\% on average).

Perceived quality of service: good evaluation by patients $(60 \%$ perceived the presence of the pharmacist in the ward, 50\% of patients claim to have received advice about treatment, in $58 \%$ of cases the pharmacist had played an important role in counselling during hospitalisation) and good evaluation by health professionals (the pharmacist had a role in informing the drug (score 3.7 on a numerical scale from 1 to 4 ) according to medical opinion and he had a role (score 3.6) in preparation and conservation, based on nurses' opinions).

Apart from the numerical results, the relevance of the project was to define a potential model of the ward pharmacist adapted to the Italian situation.

This project represents a first step in the evolution of the hospital pharmacist's role which is now required to be an integral part of the healthcare team in order to support both the management of therapies and patient safety.

\section{THE OUTPATIENT PHARMACEUTICAL SERVICES}

Dr Ilaria Uomo will describe the role and tasks of the outpatient pharmaceutical services, the twin side of the hospital pharmacy of the public health system.

The Italian Health System operates through local health authorities which manage nearly $80 \%$ of small public hospitals. Pharmaceutical services are under the control of the local health units and are responsible for various matters related to drugs and medical devices, at the interface between the hospital and the community

Educational requirements for pharmacists in the outpatient pharmaceutical services are the same as for hospitals (eg, they need hospital pharmacy specialisation).

Their activities can be classified as: (a) towards patients: drug information, dispensing of specialist drugs; (b) towards general practitioners: prescription monitoring, as a guarantee of compliance with the Italian Medicine Agency guidelines; prescription surveillance as a measure of cost containment, in cooperation with various levels of institutions (local or regional); (c) towards community pharmacies: supervision (eg, certifications and payments for all drugs dispensed free of charge under the National Health Service regulations).

The pharmaceutical services are involved in larger processes or strategies with the aims of coordinating health services and professionals, building an ideal bridge between hospitals, community districts and territorial health structures (such as family counselling, primary care physicians, emergency medical services), preserving quality of life and reducing the costs of inappropriate treatments or hospitalisations.

As required by the programmes of the school of specialisation in hospital pharmacy, the pharmaceutical services are also involved in student on the job training.

'Dipartimento Farmaceutico, ASP Palermo

'Laboratorio SIFO di Farmacoeconomia, ESTAV Centro, Prato (FI)

${ }^{3}$ Ospedale Fratelli Crobu , ASL di Carbonia (CI)

${ }^{4}$ Università degli Studi di Bologna

${ }^{5}$ Servizio di Farmacia, AOU San Giovanni Battista, Torino

${ }^{6}$ Servizio Farmaceutico, Asl 4 Terni

${ }^{7}$ Servizio di Farmacia, AOUI Verona

* SIFO Member

§SIFO Delegation for EAHP Coordinator

- SIFO Board Member and Scientific Committee

Coordinator

Competing interests None.

Provenance and peer review Commissioned; not externally peer reviewed. 